\title{
Blood eosinophil count and exacerbation risk in patients with COPD
}

\author{
To the Editor:
}

Exacerbations in chronic obstructive pulmonary disease (COPD) are major contributors to worsening lung function, impaired quality of life, emergency healthcare use and COPD-related mortality. COPD exacerbations are heterogeneous in terms of airway inflammation and aetiology. About $60 \%$ of COPD patients have blood eosinophil counts of $\geqslant 2 \%$, which appears to be associated with an increased risk of exacerbations [1-3]. However, the association between blood eosinophil counts at stable disease and COPD exacerbations in the subsequent year is incompletely understood.

This historical follow-up study used longitudinal medical record data to evaluate blood eosinophil counts as a biomarker of exacerbation risk by assessing the association between blood eosinophil levels measured when COPD was stable and the exacerbation rate in the subsequent year, in a broad COPD population. We also investigated whether the association differed significantly between specific patient subgroups defined by smoking status, gender, disease severity (Global Initiative for Chronic Obstructive Lung Disease (GOLD) groups A-D), or treatment with inhaled corticosteroids (ICS). Data were extracted from March 1994 to February 2014 from the United Kingdom Optimum Patient Care Research Database.

We evaluated patient characteristics during one baseline year before the index date, defined as the date of most recent eosinophil count at stable COPD with $\geqslant 1$ follow-up year available. Comorbidities included as potential confounding factors were defined as those ever recorded during the registration period, and assessed at index date. Eligible patients were aged $\geqslant 40$ years, had a recorded COPD diagnosis, no other chronic respiratory disease, including no asthma-related code ever recorded, with at least one blood eosinophil count measured at stable disease (no COPD exacerbations within previous or subsequent 4 weeks). Other eligibility criteria were forced expiratory volume in $1 \mathrm{~s}$ (FEV1)/forced vital capacity (FVC) $<0.70$ recorded within 5 years of index date, history of cigarette smoking (current and ex-smokers) and $\geqslant 1$ year of data before and after index date. Eosinophil counts ranging from $0.05 \times 10^{9} / \mathrm{L}$ to $<0.45 \times 10^{9} / \mathrm{L}$ were the reference category. Low counts were $<0.05 \times 10^{9} / \mathrm{L}$, and elevated counts $\geqslant 0.45 \times 10^{9} / \mathrm{L}$, based on reference values applied in UK laboratories $\left(0.04-0.40 \times 10^{9} / \mathrm{L}\right.$ for adults) [4]. Analyses were adjusted for sex, age, body mass index, smoking status, therapy and history of comorbidities associated with eosinophil counts. COPD exacerbations were defined as: unscheduled hospital admission or emergency department attendance, and/or an acute course of oral corticosteroids, and/or antibiotics prescribed for a lower respiratory consultation. The association of blood eosinophil counts with subsequent exacerbations was analysed by estimating rate ratios (RRs) with $95 \%$ confidence intervals (CIs) using a quasi-Poisson regression model allowing for over-dispersion [5].

Of 64,847 identified patients with COPD, 8318 were eligible for inclusion in the study (56\% men and mean \pm SD age of $70 \pm 10$ years). Modified Medical Research Council (mMRC) scores were available for 6660 of these patients, for determining GOLD groups [6]. During the follow-up year, $40 \%$ of patients with reference eosinophil counts, $42 \%$ with elevated counts, and $43 \%$ with low counts experienced $\geqslant 1$ COPD exacerbations, whereas $16 \%, 18 \%$, and $17 \%$, respectively, experienced $\geqslant 2$ COPD exacerbations. Overall, patients with elevated blood eosinophil counts $(8.9 \%)$ had a $13 \%$ higher exacerbation rate during the following year than patients in the reference group $(87.7 \%)$. When investigating this association in different patient subgroups, we found a significant difference between ex-smokers and current smokers, with ex-smokers showing the higher exacerbation rate in patients with elevated blood eosinophil counts (table 1). We further compared exacerbation rates in four different patient subgroups, defined by smoking

@ERSpublications

Blood eosinophil count may serve as a biomarker of exacerbation risk in subgroups of patients with COPD http://ow.ly/6tm530cEAeO

Cite this article as: Kerkhof M, Sonnappa S, Postma DS, et al. Blood eosinophil count and exacerbation risk in patients with COPD. Eur Respir J 2017; 50: 1700761 [https://doi.org/10.1183/13993003.007612017]. 
TABLE 1 Rate ratios for the association between elevated blood eosinophil count and chronic obstructive pulmonary disease (COPD) exacerbation during follow-up year in the total population of COPD patients and in subgroups of patients defined by gender, smoking status, inhaled corticosteroid (ICS) therapy and Global Initiative for Chronic Obstructive Lung Disease (GOLD) group. The rate of COPD exacerbation in patients with an elevated eosinophil count $\left(\geqslant 0.45 \times 10^{9}\right.$ per L) relative to patients with a reference eosinophil count $\left(0.05 \times 10^{9}\right.$ per $L$ to $<0.45 \times 10^{9}$ per $\left.L\right)$ is shown for each subgroup. Differences between subgroups are tested by including an interaction term of elevated eosinophil count and the variable used to define the categories of the subgroup in a multiple regression model (e.g. interaction term of elevated eosinophil count (yes/no) $\times$ gender (male/female) had a p-value of 0.11 .

\begin{tabular}{|c|c|c|c|c|c|}
\hline Study population & Subjects & Elevated eosinophil count & Rate ratio $(95 \% \mathrm{Cl})^{\#}$ & p-value & $\mathrm{p}$-value interaction \\
\hline Male & $4695(56.4)$ & 11.6 & $1.21(1.06-1.38)$ & 0.005 & 0.11 \\
\hline Current smokers & $3610(43.4)$ & 8.7 & $0.86(0.71-1.05)$ & 0.14 & 0.0002 \\
\hline Ex-smokers & $4708(56.6)$ & 9.1 & $1.32(1.15-1.51)$ & $<0.0001$ & \\
\hline $\mathrm{ICS}^{\text {ๆ }}$ & $4082(49.1)$ & 9.4 & $1.17(1.02-1.35)$ & 0.03 & 0.29 \\
\hline A & $2357(35.4)$ & 8.2 & $0.99(0.77-1.29)$ & 0.97 & reference \\
\hline$B$ & $1364(20.5)$ & 8.8 & $1.33(1.02-1.73)$ & 0.04 & 0.07 \\
\hline C & $1379(20.7)$ & 7.9 & $1.27(0.99-1.63)$ & 0.06 & 0.12 \\
\hline $\mathrm{D}$ & $1560(23.4)$ & 9.6 & $1.17(0.95-1.44)$ & 0.13 & 0.24 \\
\hline
\end{tabular}

Data are presented as \% or $\mathrm{n}(\%)$ unless otherwise stated. Bold values indicate significant effects. ICS: inhaled corticosteroids; GOLD: Global Initiative for Chronic Obstructive Lung Disease; FEV1: forced expiratory volume in $1 \mathrm{sec}$; FVC: forced vital capacity; MRC: Medical Research Council. \#: adjusted for potential confounders; ๆ: maintenance treatment with ICS; ${ }^{+}$: GOLD groups (where data is available): for patients with FEV $/ F V C<0.70$, defined based on an MRC score $\geqslant 2$ (yes, B or $D ;$ no, A or $C$ ), number of baseline exacerbations $\geqslant 2$ or leading to hospitalisation $\geqslant 1$ or $\mathrm{FEV}_{1}$ (\% predicted) $<50 \%$ (yes, $\mathrm{C}$ or $\mathrm{D}$; no, A or B). Obstruction defined as FEV $1 / \mathrm{FVC}<0.70$ at spirometry measurement closest to index date within $\leqslant 5$ years.

status and eosinophil counts. The reference group comprised ex-smokers with eosinophil counts in the reference range $\left(0.05-0.45 \times 10^{9} / \mathrm{L}\right)$. Ex-smokers with elevated eosinophil counts had the highest exacerbation rate (RR 1.30; 95\% CI 1.14-1.48); current smokers with elevated eosinophil counts had the lowest exacerbation rate (RR $0.89 ; 95 \%$ CI $0.73-1.08$ ). We also found significant associations between elevated eosinophil counts and exacerbation rates in men (RR 1.21; 95\% CI 1.06-1.38), patients treated with ICS (RR 1.17; 95\% CI 1.02-1.35), and patients in GOLD group B (table 1). However, there was no significant effect modification by any of these characteristics. Further analysis of associations within the ex-smokers subgroup showed a significant increase in exacerbation rate with elevated eosinophil counts in male ex-smokers ( $n=2914$; RR 1.40; 95\% CI 1.20-1.64), ex-smokers treated with ICS ( $n=2545$; RR 1.37; 95\% CI 1.17-1.62) and ex-smokers in GOLD groups B-D (table 1). However, there was no significant effect modification by these characteristics. For COPD patients with low eosinophil counts (3.4\%), the exacerbation rate was not significantly different from that in patients with reference counts (RR 1.06; 95\% CI 0.89-1.27).

We show that an elevated blood eosinophil count $\left(\geqslant 0.45 \times 10^{9} / \mathrm{L}\right)$ in patients with stable COPD is associated with a higher exacerbation rate the following year. We observed that: (i) the increased exacerbation rate with elevated eosinophil count was restricted to ex-smokers; (ii) this association was significant both in male ex-smokers and in patients on ICS, but there was no interaction effect between sex or ICS and eosinophil count on exacerbation rate; (iii) patients with an elevated eosinophil count who were currently smoking showed the lowest exacerbation rate; and, (iv) there was no association between a low eosinophil count and COPD exacerbations.

A recent observational study $(\mathrm{n}=7225)$ reported that blood eosinophil counts $>0.34 \times 10^{9} / \mathrm{L}$ are associated with increased risk of moderate and severe exacerbations in patients with spirometrically confirmed COPD [7]. However, the study did not investigate other patient characteristics that might affect the association between blood eosinophils and exacerbations. Our findings suggest that ex-smokers with stable COPD and elevated blood eosinophil counts may constitute a target population for more specific treatment of eosinophilic inflammation, because the increased risk was more pronounced in patients receiving ICS and this treatment does not appear to lower the blood eosinophil count [8]. Our finding of a higher percentage of men than women having elevated eosinophil counts is consistent with that of the Copenhagen study [7]. Gender-related differences in airway dimensions, structure, and function, together with variations in 
pathophysiological and, more specifically, inflammatory mechanisms elicited by tobacco smoking, may lead to differences in clinical manifestations of airway disease [9-11]. There is also some evidence that active tobacco smoking has a suppressive effect on eosinophils and inflammatory cells or cytokines [12], even in intermittent smokers [13].

We acknowledge that our study population is broader than the COPD population classically included in clinical trials. Real-life patients include a diverse spectrum of individuals with physician-diagnosed COPD. Many of these patients would not meet eligibility criteria for randomised controlled trials [14, 15], but are representative of the patients seen by clinicians. Study strengths include the large patient population drawn from geographically and socioeconomically diverse practices throughout the UK. In addition, $85 \%$ of our patients had recent spirometry results; GOLD groups could be defined in $80 \%$; and $81 \%$ had blood count measurements. There are some limitations: i) although we used eosinophil counts at stable disease, we cannot exclude the possibility that specific indications may have influenced blood eosinophil levels, leading to an underestimation of the association between eosinophils and exacerbations; ii) we studied only the association with exacerbation rate in the subsequent year and did not assess whether there is a sustained increased risk over time; iii) we used the mMRC dyspnoea scale score to assign GOLD groups and results may have differed had we used the COPD assessment test [16].

To conclude, we have shown that elevated blood eosinophil counts may predict COPD exacerbation risk only in ex-smokers. This analytical strategy may eventually help in the targeting of therapy to specific patient subgroups to maximise benefit and minimise risk for individual patients.

Marjan Kerkhof ${ }^{1}$, Samatha Sonnappa ${ }^{1}$, Dirkje S. Postma ${ }^{2}$, Guy Brusselle ${ }^{3,4,5}$, Alvar Agusti ${ }^{6,7}$, Antonio Anzueto ${ }^{8,9}$, Rupert Jones ${ }^{10}$, Alberto Papi $\circledast^{11}$, Ian Pavord ${ }^{12}$, Emilio Pizzichini ${ }^{13}$, Todor Popov ${ }^{14}$, Nicolas Roche ${ }^{15,16}$, Dermot Ryan $^{17}$, Mike Thomas ${ }^{18,19}$, Claus Vogelmeier ${ }^{20,21}$, Alison Chisholm ${ }^{22}$, Daryl Freeman ${ }^{23}$, Mona Bafadhel ${ }^{12}$, Elizabeth V. Hillyer ${ }^{1}$ and David B. Price $\oplus^{1,22,24}$

${ }^{1}$ Observational and Pragmatic Research Institute (OPRI), Singapore, Singapore. ${ }^{2}$ Dept of Pulmonology, University Medical Center Groningen, University of Groningen, Groningen, The Netherlands. ${ }^{3}$ Dept of Respiratory Medicine, Ghent University Hospital, Ghent, Belgium. ${ }^{4}$ Dept of Epidemiology, Erasmus Medical Center, Rotterdam, The Netherlands. ${ }^{5}$ Dept of Respiratory Medicine, Erasmus Medical Center, Rotterdam, The Netherlands. ${ }^{6}$ Respiratory Institute, Hospital Clínic, Institut d'Investigacions Biomèdiques August Pi i Sunyer (IDIBAPS), University of Barcelona, Barcelona, Spain. ${ }^{7}$ Centro de Investigación Biomédica en red Enfermedades Respiratorias (CIBERES), Madrid, Spain.

${ }^{8}$ Division of Pulmonary Diseases and Critical Care Medicine, School of Medicine, The University of Texas Health Science Center, San Antonio, TX, USA. ${ }^{9}$ Audie L. Murphy Memorial VA Hospital, South Texas Veterans Health Care System, San Antonio, TX, USA. ${ }^{10}$ Plymouth University Peninsula Schools of Medicine and Dentistry (PU PSMD), Plymouth University, Plymouth, UK. ${ }^{11}$ Dept of Medicine, University of Ferrara, Ferrara, Italy. ${ }^{12}$ Respiratory Medicine Unit, Nuffield Dept of Medicine, University of Oxford, Oxford, UK. ${ }^{13}$ Asthma Research Centre (NUPAIVA), University Hospital, Federal University of Santa Catarina, Florianópolis, Brazil. ${ }^{14}$ Clinic of Allergy and Asthma, Medical University Sofia, Sofia, Bulgaria. ${ }^{15}$ Unit EA2511 (Cystic Fibrosis and Chronic bronchopathy), Faculté de Médecine, Université Paris Descartes, Paris, France. ${ }^{16}$ Hôpital Cochin, Assistance Publique Hôpitaux de Paris, Paris, France. ${ }^{17}$ Allergy and Respiratory Research Group, Usher Institute of Population Health Sciences and Informatics, University of Edinburgh, Edinburgh, UK.

${ }^{18}$ Primary Care and Population Sciences, Aldermoor Health Centre, University of Southampton, Southampton, UK.

${ }^{19}$ NIHR Southampton Respiratory Biomedical Research Centre, University Hospital Southampton NHS Foundation Trust, Southampton, UK. ${ }^{20}$ Dept of Medicine (Pulmonary and Critical Care Medicine), University Medical Center Giessen and Marburg, Philipps-University Marburg, Marburg, Germany. ${ }^{21}$ German Center for Lung Research (DZL), Marburg, Germany. ${ }^{22}$ On behalf of the Respiratory Effectiveness Group, Cambridge, UK. ${ }^{23}$ Mundesley Medical Centre, Mundesley, UK. ${ }^{24}$ Institute of Applied Health Sciences (Academic Primary Care), University of Aberdeen, Aberdeen, UK.

Correspondence: DB Price, Institute of Applied Health Sciences (Academic Primary Care), University of Aberdeen, Polwarth Building, Foresterhill, Aberdeen, AB25 2ZD, UK. E-mail: dprice@opri.sg

Received: Nov 142016 | Accepted after revision: April 222017

Support statement: support was provided by the Respiratory Effectiveness Group (REG-RES1306). Funding information for this article has been deposited with the Crossref Funder Registry.

Conflict of interest: Disclosures can be found alongside this article at erj.ersjournals.com

\section{References}

1 Bafadhel M, McKenna S, Terry S, et al. Blood eosinophils to direct corticosteroid treatment of exacerbations of chronic obstructive pulmonary disease: a randomized placebo-controlled trial. Am J Respir Crit Care Med 2012; 186: $48-55$.

2 Pascoe S, Locantore N, Dransfield MT, et al. Blood eosinophil counts, exacerbations, and response to the addition of inhaled fluticasone furoate to vilanterol in patients with chronic obstructive pulmonary disease: a secondary analysis of data from two parallel randomised controlled trials. Lancet Respir Med 2015; 3: 435-442.

3 Watz $\mathrm{H}$, Tetzlaff $\mathrm{K}$, Wouters EF, et al. Blood eosinophil count and exacerbations in severe chronic obstructive pulmonary disease after withdrawal of inhaled corticosteroids: a post-hoc analysis of the WISDOM trial. Lancet Respir Med 2016; 4: 390-398.

4 UK National Health Service NHS. Full Blood Count. www.pathology.leedsth.nhs.uk/pathology/ClinicalInfo/ Haematology/FullBloodCount.aspx Date last updated: April 25, 2013. Date last accessed: June 06, 2017. 
5 Ver Hoef JM, Boveng PL. Quasi-Poisson vs. negative binomial regression: how should we model overdispersed count data? Ecology 2007; 88: 2766-2772.

6 Global strategy for the diagnosis, management and prevention of COPD. Global initiative for chronic obstructive lung disease (GOLD), 2016. http://goldcopd.org/global-strategy-diagnosis-management-prevention-copd-2016/ Date last accessed: June 06, 2017.

7 Vedel-Krogh S, Nielsen SF, Lange P, et al. Blood eosinophils and exacerbations in chronic obstructive pulmonary disease. The Copenhagen General Population Study. Am J Respir Crit Care Med 2016; 193: 965-974.

8 Barnes NC, Sharma R, Lettis S, et al. Blood eosinophils as a marker of response to inhaled corticosteroids in COPD. Eur Respir J 2016; 47: 1374-1382.

9 Becklake MR, Kauffmann F. Gender differences in airway behaviour over the human life span. Thorax 1999; 54: 1119-1138.

10 Faner R, Gonzalez N, Cruz T, et al. Systemic inflammatory response to smoking in chronic obstructive pulmonary disease: evidence of a gender effect. PLoS One 2014; 9: e97491.

11 Aryal S, Diaz-Guzman E, Mannino DM. Influence of sex on chronic obstructive pulmonary disease risk and treatment outcomes. Int J Chron Obstruct Pulmon Dis 2014; 9: 1145-1154.

12 van der Vaart H, Postma DS, Timens W, et al. Acute effects of cigarette smoke on inflammation and oxidative stress: a review. Thorax 2004; 59: 713-721.

13 van der Vaart H, Postma DS, Timens W, et al. Acute effects of cigarette smoking on inflammation in healthy intermittent smokers. Respir Res 2005; 6: 22.

14 Travers J, Marsh S, Caldwell B, et al. External validity of randomized controlled trials in COPD. Respir Med 2007; 101: 1313-1320.

15 Halpin DM, Kerkhof M, Soriano JB, et al. Eligibility of real-life patients with COPD for inclusion in trials of inhaled long-acting bronchodilator therapy. Respir Res 2016; 17: 120.

16 Karloh M, Fleig MA, Maurici R, et al. The COPD assessment test: what do we know so far? A systematic review and meta-analysis about clinical outcomes prediction and classification of patients into GOLD stages. Chest 2016; 149: 413-425. 